\title{
Telelactation with a Mobile App: User Profile and Most Common Queries
}

\section{Telelactation with a Mobile App}

Padró-Arocas, Alba ${ }^{1}$

Mena-Tudela, Desirée ${ }^{2}$

Baladía, Eduard ${ }^{3}$

Cervera-Gasch, Agueda ${ }^{2}$

González-Chordá, Víctor Manuel²

Aguilar-Camprubí, Laia ${ }^{4}$

${ }^{1}$ International Board Certified Lactation Consultant (IBCLC).

${ }^{2}$ PhD. Department of Nursing, Faculty of Health Sciences. Universitat Jaume I (Castellón, Spain)

${ }^{3}$ Bachelor of Science in Human Nutrition and Dietetics. Evidence-Based Nutrition Network (RED-NuBE), Spanish Academy of Nutrition and Dietetics (AEND), 31006 Navarra, Spain

${ }^{4}$ Midwifery. Atenció a la Salut Sexual i Reproductiva (ASSIR) Esquerra, Institut Català de la Salut, Sabadell, Barcelona, Spain

\section{Corresponding author:}

Desirée Mena-Tudela. Department of Nursing. University Jaume I. Avda. Sos I Baynat s/n 12071, Castellón de la Plana, Spain. dmena@uji.es

Funding Statement: The authors received no financial support for the research, author-ship, and/or publication of this article. 


\title{
Telelactation with a Mobile App: User Profile and Most Common Queries
}

\begin{abstract}
Background: Mobile applications related to health issues are currently expanding. Different uses of new technologies have produced positive results regarding breastfeeding support. Breastfeeding applications are increasing.

Objective: We conducted a descriptive analysis of a mobile application for breastfeeding (LactApp) to study the user profile and the most frequent queries.

Materials and Methods: This was a retrospective, comparative and descriptive ecological time series study of LactApp from 2016 to 2019. Google Analytics and the app itself were used for data collection. The data were analysed in Excel, and for the time series, Prais-Winsten auto-regressions were applied based on the Durbin-Watson method in Stata.
\end{abstract}

Results: A total of 115,830 users and 71,780 infants were registered in the application. A total of $1.91 \%$ of these users obtained the medical version. The application was used for both queries and surveys and for users to interact through chat. A total of $30.17 \%$ of the responses were related with "baby's sleep" $(8.94 \%), 8.91 \%$ were related to "preservation of milk", $6.16 \%$ were related to "breastfeeding crisis", and $6.15 \%$ were related to "physiological evolution of breastfeeding", all with an increasing trend.

Conclusion: LactApp is a resource for breastfeeding that is widely downloaded and used by a substantial number of individuals. The most recurring topics were baby's sleep, milk extraction and preservation, breastfeeding crisis and physiological evolution of breastfeeding. 


\section{Introduction}

The scientific literature states that infants who are breastfed and mothers who breastfeed are exposed to many health benefits, both in the short term and long term. The World Health Organization (WHO), the United Nations Children's Fund (UNICEF) and other international organizations recommend initiating breastfeeding (BF) during the first hour of life of the newborn and exclusively BF up to 6 months of age, subsequently introducing adequate complementary foods without stopping BF until the child is at least 2 years old ${ }^{1}$. The rates of $\mathrm{BF}$ at the global level do not conform to these recommendations; each year, approximately 823,000 children's lives could be saved if the infants were breastfed ${ }^{2}$, and only $40 \%$ of infants younger than 6 months receive human milk as exclusive food ${ }^{3}$. Support, counselling and education are fundamental strategies for a mother to continue BF because it is a bio-socio-cultural act for women, although it is instinctive for the newborn ${ }^{4}$.

Interventions like as telephone calls ${ }^{5}$, short messages $(\mathrm{SMS})^{6}$, telelactation techniques ${ }^{7}$ and online forums ${ }^{8}$ have shown positive efficacy in establishing and maintaining $\mathrm{BF}$, noting that women increasingly use the Internet to stay updated about $\mathrm{BF}^{9}$. The development of mobile applications related to health issues has been increasing in recent years ${ }^{10}$. This increase has also occurred with mobile applications (apps) related to BF, with a progressive increase in the launch of apps for BF from 2011 to the present; in 2016 and 2017 there was a striking peak in the launch of these apps ${ }^{11}$. The number of app downloads is also $\operatorname{high}^{11,12}$, which indicates a new method for mothers to seek support and information related to BF. With the intention of increasing BF rates, transferring the marketing and promotion techniques used by artificial milk companies to promote BF may be beneficial, and making use of new technologies, social media and mobile phones may generate positive results ${ }^{13}$. 
One of these apps is LactApp, which was developed in Spain and has been in use since 2016. This tool is accessible 24 hours a day with an Internet connection, providing personalized and convenient support using artificial intelligence. LactApp functions as a selfadministered questionnaire based on more than 50 decision trees constructed with questions and answers developed by professional breastfeeding experts, backed by scientific evidence and updated with official health recommendations. The result of the questionnaire provides more than 2,300 personalized responses that can be reached through more than 76,100 possible paths, which vary according to the profile of the user and the options that they select. This allows data sampling through an ecological momentary assessment ${ }^{14}$. To arrive at a final response in the questionnaire, the user must have registered on the application and provided their e-mail address and then navigate through the options of the self-administered questionnaire until reaching a final response. The research aim of this study was to perform a descriptive analysis of LactApp to study the profile of users as well as the most frequent queries.

\section{Methods}

\section{Design}

A retrospective, comparative and descriptive ecological study of time series data recorded by the LactApp tool from 01.07.2016 to 30.06.2019. This study complied with Organic Law 3/2018, of December 5, on the Protection of Personal Data and guarantee of digital rights. Registration in LactApp requires users to accept Regulation (EU) 2016/679 of the European Parliament and of the Council of 27 April 2016 regarding the protection of individuals with respect to the processing of personal data and the free circulation of these data (General Regulation of Data Protection).

\section{Setting}


The LactApp tool is a mobile application dedicated to BF. LactApp was developed by the authors of the study. The main functionality of LactApp is its automated breastfeeding query system. It also provides features related to lactation monitoring, like as a record of breastfeeding sessions, where the user can record the number of and time for daily BF sessions and difficulties related to BF (pain, cracks, lumps, colour changes in the nipple, degree of pain sensation, and mood). The weight, height, and bowel movements of the infant can also be recorded.

Other functionalities that LactApp currently has are breastfeeding surveys and personalized plans. The former comprise 5 questionnaires that allow for a quick response in relation to situations that a mother can experience during breastfeeding (The first 5 days with your baby; Successfully completing the first month; Does my baby breastfeed well?; Is my baby ready to eat solids?; Do you know if your baby has a short tongue frenulum?). Personalized plans involve questionnaires that use propositional logic with several variables for proposals and recommendations to users based on their needs and preferences.

In addition to the self-administered questionnaire, it is also possible to consult or comment on BF questions through a live chat feature hosted by an IBCLC (International Board Certified Lactation Consultant) expert on the LactApp team (implemented in the first trimester of 2018). The flow of queries and interactions through chat allows the accumulation of artificial intelligence for the machine learning and case-based reasoning used by the app. The analysis process is based on Support Vector Machine techniques ${ }^{15}$ a supervised learning model used for classification. Being a supervised model, Support Vector Machines need a set of labelled data for training the system and "teaching" it to perform the required task. In order to facilitate this labelling process our experts use a custom solution that allows them to label queries while providing a valid reply for the user. Labels are based on custom classification trees, we are currently deploying an ontologic solution to add semantics to such trees ${ }^{16}$ 
(Figure 1). LactApp has 50 query topics related to BF, maternity, maternal and infant health and pregnancy, among others, divided into large query areas.

In addition to the app, LactApp also has social channels, like as the LactApp blog, Instagram, Facebook and YouTube, to disseminate information regarding $\mathrm{BF}$ and receive comments and inquiries. Finally, this application has a medical version in which health professionals can access all the available content of the application involving a response as a function of a query made by the professional, for example, the age of the infant involved in the consultation. LactApp can be downloaded from Android and iOS. Users need to log in following the instructions. If the user wishes to obtain a Medical profile, they must indicate this in their profile. LactApp is available in Spanish and English.

\section{Sample}

The study population consisted of all LactApp users. As this is an ecological study with aggregated data, it was not necessary to carry out a sample size calculation, as the study was based on the trend of usage indicators in Google Analytics.

\section{Measurement}

Google Analytics was used for measurement and data collection. Google Analytics is a web analytics tool that can provide with basic information. This tool tracks usage of the web or an app by sending a traking label that indicates that the page has been displayed and because the tracking is collected by Google, the website operator a more complete picture of user behaviour ${ }^{17}$.

\section{Data collection}

Google Analytics does not contain any personal data or confidential information and is presented as aggregated data ${ }^{17}$. People who enter and register at LactApp accept the legal 
and data protection conditions stated above. Data extraction was performed by trimesters to compare data trends. Among the variables collected with Google Analytics were number of users, number of sessions and screens, duration of the session, new users and recurring users, access languages, countries from which the app was accessed, operating system used and types of events: number of queries and topics, chat access and surveys performance. Unique events, defined by Google Analytics as interactions with content by a single user within a single session ${ }^{18}$, were taken into account for the events. In addition, registration in LactApp by the users allows the collection of sociodemographic data like as age, gender, number of children, country, academic degree and profession (medical version).

\section{Data analysis}

A descriptive analysis was performed for all variables, using the absolute and relative frequencies expressed in percentages for qualitative variables and the mean and standard deviation for quantitative variables. In addition, the temporal trend of the queries performed in the application was estimated by performing Prais-Winsten auto-regressions based on the Durbin-Watson method. This analysis allows obtaining the global trend of the quantitative variables related to specific and studied moments according to their distribution over time. Thus, the usage trend was determined (increasing, decreasing or static) by calculating the 3month percent change (TPC) and its $95 \%$ confidence interval $(95 \% \mathrm{CI})^{19}$. The analysis was performed using Excel spreadsheets for general calculations and Stata 14.0 software for time series (2015).

\section{Results}

A total of 115,830 users were registered in the LactApp application in the study period (from 01.07.2016 to 30.06.2019). Of these, $1.91 \%(n=2,207)$ obtained the medical version of the application. The mean age of the users of the medical version was 34.57 years 
$(S D=6.02$ years $)$ and the $85.76 \%$ of the others users was between 25-44 years. Sociodemographic data are shown in Table 1.

In the same period, a total of 71,780 infants were registered. Of these, $49.4 \%(n=$ $35,446)$ were female and $50.6 \%(n=36,334)$ were male. The median age of the registered infants was 21 months $(I Q R=14$ months; minimum $=0$, maximum $=78)$. The mean weight of infants at birth was 3,211.3 grams ( $S D=498.17$ grams), with an average height of 49.61 centimetres $(S D=2.42$ centimetres). A total of $4,388(6.1 \%)$ of the infants were premature, born between weeks 25 and 36 of gestation, with a mean of 34.73 weeks ( $S D=2.05$ weeks).

During the study period, a total of 1,767,308 sessions were initiated, with a significant increasing trend and a total of $11,838,570$ screens were viewed, with a static trend. The average number of screens consulted per session was 6.7 , with a significant decreasing trend and an average duration of 6 minutes and 2 seconds per session, which did not decrease in a significant way in the period studied. The percentage of recurrent users increased significantly in this period and new users decreased. Table 2 shows these data distributed by trimester.

The distribution of countries by percentage of overall LactApp users was as follows: Spain $(53.84 \%, n=62,363)$, Mexico $(11.35 \%, n=13,147)$; Argentina $(5.48 \%, n=6,347)$, United States $(5.30 \%, n=6,139)$, Chile $(4.84 \%, n=5,606)$, Colombia $(3.9 \%, n=4,517)$, Venezuela $(1.78 \%, n=2,062)$, Peru $(1.57 \%, n=1,818)$, Uruguay $(1.26 \%, n=1,460)$ and Costa Rica $(1.1 \%, n=1,274)$. The most consulted languages were Spanish $(89.94 \%, n=$ 112,092) and English $(5.22 \%, n=2,477)$.

Table 3 provides unique events among the most relevant events in LactApp by trimester in the study period. In the study period, users started 1 of the 5 existing surveys 62,173 times $(T P C=429.203 ; 95 \% C I=-8,903$ to 867,311 ; trend $=$ increasing $)$, of which 
71.13\% ( $n=44,222)$ got to answer completely, with an increasing trend. Regarding the opinion of the users who arrived at a final response to the surveys, $15.11 \%(n=6,680)$ provided their opinion, with $93.01 \%(n=6.215)$ clicking "Like". The survey that was most often initiated was "Does my baby breastfeed well?", with $32.32 \%(n=20,094)(T P C=$ $140.871 ; 95 \% C I=-5.184$ to 286.927 ; trend = static), followed by " Successfully completing the first month", with $18.34 \%(n=11,400)(T P C=80.817 ; 95 \% C I=1.542$ to 160.092 ; trend = growing $)$, "The first 5 days with your baby", with $17.56 \%(n=10,920)(T P C=67.956$; $95 \% C I=-9.766$ to 145.678 ; trend = static), "Is my baby ready to eat solids?", with $16.59 \%$ $(n=10,313)(T P C=73.731 ; 95 \% C I=2.550$ to $144.912 ;$ trend $=$ increasing $)$ and finally, "Do you know if your baby has a short tongue frenulum?", with $15.19 \%(n=9,446)(T P C=$ $71.174 ; 95 \% C I=3.904$ to 138.443 ; trend $=$ increasing).

Finally, in the study period, users obtained 2,757,702 final responses in LactApp. Of these responses, $1.68 \%(n=46,374)$ of users opined on the quality of the final responses, with $89.26 \%(n=41,392)$ clicking "Like" and $10.74 \%(n=4,982)$ clicking "Unlike". A total of $30.17 \%$ of the responses corresponded to the topics "baby's Sleep" ( $n=246,628 ; 8.94 \%)$, "preservation of milk" ( $n=245,785 ; 8.91 \%)$; "breastfeeding crisis" ( $n=169,911 ; 6.16 \%)$, and "physiological evolution of breastfeeding" ( $n=169,562 ; 6.15 \%)$ (Table 4, Supplemental file 1). Specifically, the most consulted final responses by each topic are shown in Table 5.

\section{Discussion}

This study aimed to describe the queries and interactions of users of a mobile application for BF (LactApp) in order to study both the user profile and the most frequent queries. Notably, the large number of users of LactApp as well as the growing number of queries suggests that it is a very relevant way of consulting for maternal support regarding breastfeeding. 
According to the mHealth Economics report ${ }^{20}$, downloads of health-related applications have been growing exponentially since 2013. Taking into account the figures provided by this report, LactApp obtained more active users than $83 \%$ of apps, being among the $20 \%$ most downloaded apps and active users in the world. It is important to highlight that $60.94 \%$ of LactApp's Medical version, are health professionals who, perhaps, in search of information or in the form of support in this professional field make use of LactApp, given the lack of training and support that, in general, health professionals receive in $\operatorname{Spain}^{21}$ and at the international level ${ }^{22,23}$. Future studies will help to confirm whether LactApp is a useful resource for professionals to make decisions on breastfeeding.

Internet users increasingly obtain more information through smartphones ${ }^{24}$; therefore, the use of apps in the field of $\mathrm{BF}$ is gaining ground and becoming a pillar in the establishment and subsequent maintenance of $\mathrm{BF}^{25}$. This globalized fact can explain the growing trend in queries in LactApp. The static trend regarding the number of screens visited by the users and the duration of each session, in addition to the decreasing trend of the average number of screens consulted, may be related to the improvements implemented in LactApp throughout the studied time period. Improvements that stand out are those to the interface, revitalisation of queries and reorganization of content. Currently, from the initial screen you can see the thematic areas of each section of LactApp. In addition, the blog no longer has a separate button but is accessible from the initial screen. Finally, a tab bar has been added and is always visible, so that it is faster to navigate through LactApp and get, for example, to the home page. Therefore, we believe that further research is needed to determine the usefulness of LactApp, its usability and the support it can provide to both mothers and health professionals.

Regarding the users, an advantage of LactApp is the ability to have personalized information $^{26}$ related to BF 24 hours a day, a fact that both mothers ${ }^{27}$ and health 
professionals $^{28}$ point out as an essential issue in the maintenance of BF. This fact can be related to the increasing number of users within the app and with the number of recurring users increasingly growing. It must be taken into account that the decreasing number of new users may be due to the rapid diffusion of LactApp among mothers and health professionals; since the extreme initial growth, it has not been possible to capture so many new users. Regarding this aspect, users who enter the application stay and use it regularly to address their breastfeeding questions, so they become recurrent users. Analysing how recurrent users navigate through LactApp will allow, in the future, to know the maternity period in which the integration of technology is most relevant. This should be taken into account in future studies.

BF children registered in LactApp have a profile very similar to that of international statistical reports ${ }^{29}$, implying that the profiles are real. Regarding the age of the babies, it is important to emphasize that LactApp automatically updates it as days go by to be able to adapt the answers it offers to mothers according to the age of their baby. On the other hand, only $2.9 \%$ of user's report being pregnant, $79.1 \%$ having no previous children. It is possible that these results are due to the fact that it is not required to complete these data during the on boarding process. $10 \%$ of users indicate that they are not happy with the response given by the app by clicking on the do not like button, we should consider if they feel the need for more information around their query and try other ways like as accessing the chat feature in the app or by sending an email.

The most consulted topics within LactApp are related to critical issues regarding when women stop BF. The critical issues that stand out are related to the "baby's sleep", a topic that different authors note as important in relation to the establishment and maintenance of $\mathrm{BF}^{30}$. Also noteworthy is the lack of training that health professionals have regarding safe sleeping and its importance, both in the prevention of sudden infant death syndrome and with the maintenance of $\mathrm{BF}^{31-33}$. Another topic to highlight is the "preservation of milk" ${ }^{14}$ because 
it is closely related to the duration of maternity leave and the return to work of the mother, agreeing with different authors that the completion of maternal leave and reinsertion of the mother into paid work is an important issue related to stopping $\mathrm{BF}^{35-37}$. A "breastfeeding crisis" is deeply related to the maternal sensation of lack of milk, which is one of the most prominent causes for stopping $\mathrm{BF}^{38}$, and there are still gaps in how to offer effective support in these situations ${ }^{39}$. Finally, the lack of knowledge, skills and attitudes of mothers regarding $\mathrm{BF}$, i.e., the loss of the culture of $\mathrm{BF}$, is another critical point for stopping $\mathrm{BF}^{40}$. Thus, the "physiological evolution of breastfeeding" is another section in LactApp that mothers consult often. A new study hypothesis is that apart from being informed, LactApp helps women maintain BF at times when they would normally stop this type of feeding in rural ${ }^{41}$ and urban areas, but this hypothesis should be confirmed in future studies. Also the authors are exploring the possibility of linking the topics and specific queries that users make and the ages of the babies in their profiles, as well as identifying different socio-demographic variables. It will also be interesting to investigate the consultation area and the functionality of LactApp according to the established user profile. All these questions will have to be resolved in future studies.

\section{Limitations}

This is an ecological study based on aggregate data so that there is a risk of incurring ecological fallacies in the interpretation of the data as they do not consider possible variables that can influence the results ${ }^{42}$.

Additionally, data were collected with Google Analytics, and some limitations should be taken into account, like as data sampling when the volume of data traffic is greater than 500,000 sessions. Regarding of automatically update of the babies' age this fact could affect 
the results increasing the median because the mothers continued using LactApp. For register on LactApp, it is not necessary to complete all the sociodemographic data.

Finally, we must be aware that the launch of LactApp produced very high initial statistics, which may lead to a change in the statistical estimates presented in the coming years.

\section{Conclusions}

LactApp is a mobile application with powerful resources for breastfeeding and is currently widely downloaded and in use by a significant number of users. These users periodically consult the application, with the most consulted topics being those related to the baby's sleep, the extraction and preservation of milk, breastfeeding crises and the physiological evolution of BF. The increasing use of LactApp supports the need for future research on the validation of the application as well as on the degree to which the recommendations offered are correlated with the best available scientific evidence. Despite the limitations, LactApp can be a valuable tool for health professionals to learn about the issues that mothers need most support. LactApp can also be attractive to researchers and developers because there is a wide expansion in Artificial Intelligence and usability, and to organisations that advocate breastfeeding and are involved in initiatives such as the BFHI.

Conflicts of Interest: The authors declared the following potential conflicts of interest with respect to the research, authorship and/or publication of this article: AP created and designed the software. EB has personal friendly relationships with the creators of the software under investigation.

\section{References}

1. Kim SK, Park S, Oh J, Kim J, Ahn S. Interventions promoting exclusive breastfeeding up to six months after birth: A systematic review and meta-analysis of randomized 
controlled trials. Int J Nurs Stud. 2018. doi:10.1016/j.ijnurstu.2018.01.004

2. The Lancet. Breastfeeding: achieving the new normal. Lancet. 2016;387(10017):404. doi:https://doi.org/10.1016/S0140-6736(16)00210-5

3. World Health Organization. 10 facts on breastfeeding. https://www.who.int/features/factfiles/breastfeeding/en/. Published 2017. Accessed July 11, 2019.

4. Sutton M, O’Donoghue E, Keane M, Farragher L, Long J. Interventions That Promote Increased Breastfeeding Rates and Breastfeeding Duration among Women: An Umbrella Review. Dublin: Health Research Board, Dublin; 2016. https://www.hrb.ie/fileadmin/publications_files/Interventions_that_promote_increased _breastfeeding_rates_2016.pdf.

5. Patel A, Kuhite P, Puranik A, Khan SS, Borkar J, Dhande L. Effectiveness of weekly cell phone counselling calls and daily text messages to improve breastfeeding indicators. BMC Pediatr. 2018;18(1):337. doi:10.1186/s12887-018-1308-3

6. Unger JA, Ronen K, Perrier T, et al. Short message service communication improves exclusive breastfeeding and early postpartum contraception in a low- to middle-income country setting: a randomised trial. BJOG An Int J Obstet Gynaecol. 2018;125(12):1620-1629. doi:10.1111/1471-0528.15337

7. Kapinos, K; Kotzias, V; Bogen, D; Ray, K; Demirci, J; Rigas, MA; Uscher-Pines L. The Use of and Experiences With Telelactation Among Rural Breastfeeding Mothers: Secondary Analysis of a Randomized Controlled Trial. J Med Internet Res. 2019;Sep 3. doi:10.2196/13967

8. White BK, Giglia RC, Scott JA, Burns SK. How New and Expecting Fathers Engage 
With an App-Based Online Forum: Qualitative Analysis. JMIR mHealth uHealth. 2018;6(6):e144-e144. doi:10.2196/mhealth.9999

9. Alianmoghaddam N, Phibbs S, Benn C. "I did a lot of Googling": A qualitative study of exclusive breastfeeding support through social media. Women and Birth. 2019;32(2):147-156. doi:https://doi.org/10.1016/j.wombi.2018.05.008

10. Ruiz-López T, Sen S, Jakobsen E, et al. FightHPV: Design and Evaluation of a Mobile Game to Raise Awareness About Human Papillomavirus and Nudge People to Take Action Against Cervical Cancer. JMIR Serious Games. 2019. doi:10.2196/games.8540

11. Díaz Cano AI, Margaix Fontestad L, Esplugues A. Análisis descriptivo de aplicaciones móviles sobre lactancia materna. An Pediatría. 2019;21.

doi:10.1016/j.anpedi.2019.03.009

12. Balaguer Martínez JV, Valcarce Pérez I, Esquivel Ojeda JN, Hernández Gil A, Martín Jiménez MDP, Bernad Albareda M. Telephone support for breastfeeding by primary care: a randomised multicentre trial | Apoyo telefónico de la lactancia materna desde Atención Primaria: ensayo clínico aleatorizado y multicéntrico. An Pediatr. 2018;89(6):344-351. doi:10.1016/j.anpedi.2018.02.007

13. World Health Organization WPR. Protecting Children from the Harmful Impact of Food Marketing. (World Health Organization, ed.). Brisbane (Australia); 2017. https://iris.wpro.who.int/handle/10665.1/13701.

14. Runyan JD, Steinke EG. Virtues, ecological momentary assessment/intervention and smartphone technology. Front Psychol. 2015. doi:10.3389/fpsyg.2015.00481

15. Suykens J, Vandewalle J. Least Squares Support Vector Machine Classifiers. Neural Process Lett. 1999;9:293-300. doi:https://doi.org/10.1023/A:1018628609742 
16. Antoniou G, Van Harmelen F. Web ontology language: Owl. In: Heidelberg, ed. Handbook on Ontologies. Berlin: Springer; 2004:67-92.

doi:https://doi.org/10.1007/978-3-540-24750-0_4

17. Clark D, Nicholas D, Jamali HR. Evaluating information seeking and use in the changing virtual world: The emerging role of Google Analytics. Learn Publ. 2014;27(3):185-194. doi:10.1087/20140304

18. Google. Unique Events and Unique Dimension Combinations. Analytics Help. https://support.google.com/analytics/answer/7084499?hl=en. Published 2019.

19. Antunes JLF, Cardoso MRA. Uso da análise de séries temporais em estudos epidemiológicos. Epidemiol e Serviços Saúde. 2015;24(3):565-576. doi:10.5123/S1679-49742015000300024

20. Research 2 Guidance. MHealth Economics 2017/2018.; 2017.

21. Gómez Fernández-Vegue, M.; Menéndez Orenga M. Encuesta nacional sobre conocimientos de Lactancia Materna de los residentes de peditaría en España. Rev Española Salud Púbica. 2019;93(2 de agosto e201908060).

22. Gavine A, MacGillivray S, Renfrew MJ, Siebelt L, Haggi H, McFadden A. Education and training of healthcare staff in the knowledge, attitudes and skills needed to work effectively with breastfeeding women: A systematic review. Int Breastfeed J. 2017;2(12):6. doi:10.1186/s13006-016-0097-2

23. Chuisano SA, Anderson OS. Assessing Application-Based Breastfeeding Education for Physicians and Nurses: A Scoping Review. J Hum Lact. 2019;May 21. doi:10.1177/0890334419848414

24. Ditrendia. Ditrendia - Informe Mobile 2018. Madrid (Spain); 2018. 
https://ditrendia.es/informe-mobile-2018/.

25. Silva NV de N da, Pontes CM, Sousa NFC de, Vasconcelos MGL de. Tecnologias em saúde e suas contribuições para a promoção do aleitamento materno: revisão integrativa da literatura. Cien Saude Colet. 2019;24(2):589-602. doi:10.1590/141381232018242.03022017

26. Swerts, Marlies; Westhof, Ellen; Lemiengre, Joke \& Bogaerts A. The supporting role of the midwife during the first 14 days of breastfeeding: A descriptive qualitative study in maternity wards and primary healthcare. Midwifery. 2019;78:50-57. doi:10.1016/j.midw.2019.07.016

27. Robinson A, Lauckner C, Davis M, Hall J, Anderson AK. Facebook support for breastfeeding mothers: A comparison to offline support and associations with breastfeeding outcomes. Digit Heal. 2019;5:1-12. doi:10.1177/2055207619853397

28. Garner CD, Ratcliff SL, Thornburg LL, Wethington E, Howard CR, Rasmussen KM. Discontinuity of breastfeeding care: 'There's No Captain of the Ship'. Breastfeed Med. 2016;11(1):32-39. doi:10.1089/bfm.2015.0142

29. World Health Organization. Born Too Soon: The Global Action Report on Preterm Birth.; 2012. doi:http://whqlibdoc.who.int/publications/2012/9789241503433_eng.pdf

30. Bailey C, Tawia S, McGuire E. Breastfeeding Duration and Infant Sleep Location in a Cohort of Volunteer Breastfeeding Counselors. J Hum Lact. 2019. doi:10.1177/0890334419851801

31. Angal, J; Gogoi, M; Zenel, J; Elliott A. Physicians Knowledge and Practice of Safe Sleep Recommendations for Infants in South Dakota. South Dakota Med. 2019;72(8):349-353. 
32. Salm Ward TC, Kanu FA, Anderson AK. Trends and Factors Associated with Breastfeeding and Infant Sleep Practices in Georgia. J Community Health. 2018;43(3):496-507. doi:10.1007/s10900-017-0442-4

33. Moon RY, Darnall RA, Feldman-Winter L, Goodstein MH, Hauck FR. SIDS and other sleep-related infant deaths: Evidence base for 2016 updated recommendations for a safe infant sleeping environment. Pediatrics. 2016;138(5). doi:10.1542/peds.20162940

34. Becker GE, Smith HA, Cooney F. Methods of milk expression for lactating women. Cochrane Database Syst Rev. 2016. doi:10.1002/14651858.CD006170.pub5

35. Navarro-Rosenblatt D, Garmendia ML. Maternity leave and its impact on breastfeeding: A review of the literature. Breastfeed Med. 2018;13(9):589-597. doi:10.1089/bfm.2018.0132

36. Sun K, Chen M, Yin Y, Wu L, Gao L. Why Chinese mothers stop breastfeeding: Mothers' self-reported reasons for stopping during the first six months. J Child Heal Care. 2017;21(3):353-363. doi:10.1177/1367493517719160

37. Dinour LM, Szaro JM. Employer-Based Programs to Support Breastfeeding among Working Mothers: A Systematic Review. Breastfeed Med. 2017;12(3). doi:10.1089/bfm.2016.0182

38. Onat G, Karakoç H. Three spirals: Breastfeeding problems, growth spurts, and postpartum depression. Perspect Psychiatr Care. 2020;27 August. doi:https://doi.org/10.1111/ppc.12612

39. Galipeau R, Baillot A, Trottier A, Lemire L. Effectiveness of interventions on breastfeeding self-efficacy and perceived insufficient milk supply: A systematic 
review and meta-analysis. Matern Child Nutr. 2018;14(3). doi:10.1111/mcn.12607

40. Brockway M, Benzies K, Hayden KA. Interventions to Improve Breastfeeding SelfEfficacy and Resultant Breastfeeding Rates: A Systematic Review and Meta-Analysis. J Hum Lact. 2017;33(3):486-499. doi:10.1177/0890334417707957

41. Demirci J, Kotzias V, Bogen DL, Ray KN, Uscher-Pines L. Telelactation Via Mobile App: Perspectives of Rural Mothers, Their Care Providers, and Lactation Consultants. Telemed e-Health. September 2018. doi:10.1089/tmj.2018.0113

42. Borja-Aburto VH. Estudios ecológicos. Salud Publica Mex. 2000;42(6):533-538. doi:10.1590/S0036-36342000000600010 